\title{
Recherches sur le carré de la dérivée lo- garithmique de la fonction gamma et sur quelques fonctions analogues.
}

\author{
(Par Niels Nielsen, d̀ Copenhague.)
}

I. Définitions. Formules générales.

$$
\begin{aligned}
& \mathbf{P}_{\text {osons avec Gauss }\left(^{*}\right)} \\
& \qquad \Psi(x)=D_{x} \log \Gamma(x)=-C-\sum_{n=0}^{n=\infty}\left(\frac{1}{x+n}-\frac{1}{n+1}\right),
\end{aligned}
$$

où $C$ désigne la constante d'EoLER, nous aurons pour cette fonction plus commode mais intimément liée à $\Psi(x)$, savoir la fonction

$$
s_{1}(x)=-\Psi(x)-C=\sum_{n=0}^{n=\infty}\left(\frac{1}{x+n}-\frac{1}{n+1}\right)
$$

ce développement en série de puissances

$$
s_{1}(1-x)=s_{2} x+s_{3} x^{2}+s_{4} x^{3}+\cdots, \quad|x|<1,
$$

dont les coefficients se déterminent comme voici

$$
s_{n}=\frac{1}{1^{n}}+\frac{1}{2^{n}}+\frac{1}{3^{n}}+\cdots=s_{n}(1), \quad n>1,
$$

où nous avons posé pour abréger

$$
s_{n}(x)=\frac{(-1)^{n-1}}{(n-1) !} \cdot D_{x}^{n-1} s_{1}(x)=\sum_{r=0}^{r=\infty} \frac{1}{(x+y)^{n}}, \quad n>1 .
$$

La fonction $s_{1}(x)$ s'exprimera sans peine et sous forme simple comme

(*) Disquisiliones generales circa functiones a seriem infinitam, etc., $\$ 30$; Euvres, t. III. Annali di Malematica, Serie III, tomo IX. 
intégrale définie, savoir

$$
s_{1}(x)=\int_{0}^{1} \frac{t^{x-1}-1}{1-t} d t, \quad \Re(x)(*)>0,
$$

ce qui donnera aisément cette autre formule analogue

$$
s_{n}(x)=\frac{(-1)^{n-1}}{(n-1) !} \cdot \int_{0}^{1} \frac{(\log t)^{n-1} t^{x-1}}{1-t} d t, \quad \Re(x)>0 .
$$

Nous arons encore à étudier cette nouvelle fonction transcendante

$$
\sigma_{1}(x)=\frac{1}{2}\left(s_{1}\left(\frac{x}{2}\right)-s_{1}\left(\frac{x+1}{2}\right)\right)=\sum_{r=1}^{v=\infty} \frac{(-1)^{y}}{x+y},
$$

pour laquelle nous trouvons se développement en série de puissances

$$
\sigma_{1}(1-x)=\sigma_{1}+\sigma_{2} x+\sigma_{3} x^{2}+\sigma_{4} x^{3}+\cdots, \quad|x|<1,
$$

dont les coefficients se déterminent comme voici

$$
\sigma_{n}=\frac{1}{1^{n}}-\frac{1}{2^{n}}+\frac{1}{3^{n}}-\frac{1}{4^{n}}+\cdots=\sigma_{n}(1), \quad n \geqq 1,
$$

où nous avons posé pour abréger

$$
\sigma_{n}(x)=\frac{(-1)^{n-1}}{(n-1) !} \cdot D_{x}^{n-1} \sigma_{1}(x)=\sum_{x=0}^{\infty} \frac{(-1)^{\prime}}{(x+v)^{n}} .
$$

Quant aux séries numériques $\sigma_{n}$, nous aurons pour $n=1$

$$
\sigma_{1}=\log 2
$$

où le signe $\log$ désigne le logarithme népérien, et généralement pour $n>1$

$$
\sigma_{n}=\frac{2^{n-1}-1}{2^{n-1}} \cdot s_{n}
$$

La fonction $\sigma_{1}(x)$ s'exprimera aussi sous forme simple comme intégrale définie, sıvoir

$$
\sigma_{1}(x)=\int_{0}^{1} \frac{t^{x-1}}{1+t} d t, \quad \Re(x)>0
$$

(*) Ici et dans ce qui suit $\Re(x)$ désigne la partie réelle de $x$. 
ce qui donnera immédiatement cette autre formule analogue

$$
\sigma_{n}(x)=\frac{(-1)^{n-1}}{(n-1) !} \cdot \int_{0}^{1} \frac{(\log t)^{n-1} t^{x-1}}{1+t} d t, \quad \Re(x)>0 .
$$

Dans les recherches que voici je me suis proposé d'étudier des représentations intégrales d'un produit de deux des fonctions $s_{1}(x), s_{1}(1-x), \sigma_{1}(x)$ et $\sigma_{1}(1-x)$ choisies d'une manière quelconque; les intégrales ainsi obtenues se présentent toutes sous la même forme que celles figurant au second membre de (3) et de (6). De plus, je me suis proposé de donner tous les développements possibles en séries de factorielles des produits susdits.

Il est évident qu'une telle recherche est intimément liée à une recherche sur les séries purement numériques $s_{n}$ et $\sigma_{n}$. Quant à ces séries, il est bien connu que $s_{2 n}$ et $\sigma_{2 n}$ se présentent dans les séries de puissances obtenues pour $\pi \cot \pi x$ et $\pi \operatorname{coséc} \pi x$, ce qui nous permettra de déterminer à l'aide d'une puissance de $\pi$ les sommes de toutes ces séries. On aura en effet

$$
s_{2 n}=\frac{2^{2 n-1} \cdot B_{2 n-1}}{(2 n) !} \cdot \pi^{2 n}
$$

où les nombres $B_{2 n-1}$ sont les nombres rationnels de BeRnourur. Quant aux séries $s_{2 n+1}$ et $\sigma_{2 n+1}$, à l'exception de $\sigma_{1}=\log 2$, on ignore j'usqu'au présent complètement leur nature et ne connait aucune formule récursive pour leur détermination successive. Il est bien remarquable que l'on retrouve des propriétés analogues chez les sommes des puissances des valeurs réciproques des racines d'une fonction cylindrique de première espèce et de la fonction elliptique $p(x)$ de Weierstrass.

En effet, si l'exposant figurant dans la somme des puissances des valeurs réciproques des racines de $J^{\nu}(x)$ est un nombre pair, la somme en question se présente sous forme d'une fonction rationnelle du paramètre $\nu\left({ }^{*}\right)$. Pour les racines de $p(x)$ les sommes, dont l'exposant est pair, s'expriment à l'aide des deux sommes qui correspondent aux exposants 4 et $6\left({ }^{* *}\right)$. Si, au contraire, l'exposant susdit est impair, les sommes correspondantes sont complètement inconnues.

Or, dans les recherches qui nous occupent ici, nous avons besoin d'étudier

(*) Graf: Einleitung in clie Theorie der Besscl'schen Functionen, I, p. 190; Berne, 1898.

(**) Tanniry et Moun: Théorie des fonctions elliptiques, t. I, p. 176; Paris 1893. 
les séries générales $s_{n}$ et $\sigma_{n}$ pour des valeurs paires ou impaires de l'indice $n$. Cependant des rëllexions entièrement élémentaires nous conduiront à une groupe de formules qui donnent comme cas particuliers les formules récursives bien connues pour $s_{2 n}$ et $\sigma_{2 n}$, et, sans faire connaître quelque chose sur la nature de $s_{2 n+1}$ et de $\sigma_{2 n+1}$ pour $n>0$, ces formules générales suffisent pour la résolution des problèmes susdits.

II. Remarques GÉNḱRALes sur Les trols produits $s_{n} s_{p}, s_{n} \sigma_{p}$ et $\sigma_{n} \sigma_{p}$.

Appliquons maintenant la règle de $\mathrm{C}_{\mathrm{Auchy}}$ pour la multiplication de deux séries infinies mais absolument convergentes, nous aurons immédiatement cette formule

$$
s_{n} s_{p}=s_{n+p}+c_{n, p}+c_{p, n}, \quad n>1, \quad p>1,
$$

où nous avons posé pour abréger

$$
c_{n, p}=\sum_{i=2}^{\nu=\infty} \frac{1}{v^{n}}\left(\frac{1}{1^{p}}+\frac{1}{2^{p}}+\cdots+\frac{1}{(\nu-1)^{p}}\right), \quad n>1, \quad p \geqq 1 .
$$

De la même manière nous obtiendrons, en appliquant pour $p=1$ un théoreme de M. Mertens (*), cette autre formule

$$
s_{n} \sigma_{p}=\sigma_{n+p}+d_{n, p}-\gamma_{p, n}, \quad n>1, \quad p \geqq 1,
$$

où nous avons posé

$$
\begin{gathered}
d_{n, p}=\sum_{v=2}^{v=\infty} \frac{1}{v^{n}}\left(\frac{1}{1^{p}}-\frac{1}{2^{p}}+\cdots+\frac{(-1)^{\nu}}{(v-1)^{p}}\right), \quad n>1, \quad p \geqq 1 \\
\gamma_{n, p}=\sum_{\nu=2}^{r=\infty} \frac{(-1)^{\nu}}{v^{n}}\left(\frac{1}{1^{p}}+\frac{1}{2^{p}}+\cdots+\frac{1}{(v-1)^{p}}\right), \quad n \geqq 1, \quad p \geqq 1 .
\end{gathered}
$$

Enfin nous aurons, en appliquant pour $n=p=1$ un théoreme de M. Prinasheim (**), cette troisième formule

$$
\sigma_{n} \sigma_{p}=s_{n+p}-\delta_{n, p}-\delta_{p, n}, \quad n \geqq 1, \quad p \geqq 1,
$$

(*) Journal de Crelle, t. 79 .

$(*:)$ Mathematische Annalen, t. 21. 
où nous avons posé pour abréger

$$
\delta_{n, p}=\sum_{v=2}^{p=\infty} \frac{(-1)^{\nu}}{v^{n}}\left(\frac{1}{1^{p}}-\frac{1}{2^{p}}+\cdots+\frac{(-1)^{p}}{(v-1)^{p}}\right), \quad n \geqq 1, \quad p \geqq 1
$$

mettons dans (9) $p=q=1$, nous trouvons cette formule particulière

$$
\delta_{1,1}=\frac{\pi^{2}}{12}-\frac{1}{2}(\log 2)^{2} \text {. }
$$

Il est évident que les quatre séries numériques à deux indices que nous venons d'introduire dans les expressions données pour les produits $s_{n} s_{p}, s_{n} \sigma_{p}$ et $\sigma_{n} \sigma_{p}$ jouent un rôle fondamental dans les recherches ultérieures sur ces produits. Or, il est très facile d'établir une foule d'autres formules contenant les séries $c_{n, p}, d_{n, p}, \gamma_{n, p}$, et $\delta_{n, p}$. Ici nous nous bornerons à indiquer quelques applications de la règle de décomposition de LAGrange $\left(^{*}\right)$, savoir la formule

$$
\left.\begin{array}{c}
\frac{1}{x^{p}(x-a)^{2}}=(-1)^{q} \cdot \sum_{\gamma=0}^{\nu=-1}\left(\begin{array}{c}
q+\nu-1 \\
q-1
\end{array}\right) \cdot \frac{1}{x^{p-\nu} a q+^{\nu}}+ \\
+\sum_{\nu=0}^{r=q-1}\left(\begin{array}{c}
p+\nu-1 \\
p-1
\end{array}\right) \cdot \frac{(-1)^{\nu}}{a^{p+\nu}(x-a) c^{\nu}},
\end{array}\right\}
$$

où $p$ et $q$ désignent deux positifs entiers.

En effet, mettons dans $(\alpha) x=n$ et $a=1,2,3, \ldots, n-1$, puis ajoutons toutes les équations ainsi obtenues prises avec des signes alternés, nous obtenons sans peine ces deux formules

$$
\begin{aligned}
& \gamma_{p, q}=(-1)^{q} \cdot \sum_{\nu=0}^{\nu=p-1}\left(\begin{array}{c}
q+\nu-1 \\
q-1
\end{array}\right) \gamma_{p-\nu, q+\nu}+ \\
& +\sum_{v=0}^{v=q-1}(-1)^{\nu}\left(\begin{array}{c}
p+\nu-1 \\
p-1
\end{array}\right) \sigma_{p+\nu} \cdot \sigma_{q-\nu} \\
& \left.\begin{array}{c}
d_{p, q}=(-1)^{q} \cdot \sum_{\nu=0}^{r=p-1}\left(\begin{array}{c}
q+\nu-1 \\
q-1
\end{array}\right) \delta_{p-\eta, q+\nu}+ \\
+\sum_{\nu=0}^{\gamma=q-1}(-1)^{\nu}\left(\begin{array}{c}
p+\nu-1 \\
p-1
\end{array}\right) s_{p+\nu \cdot \sigma_{q-\nu}}
\end{array}\right\}
\end{aligned}
$$

dans (11) il faut admettre $p>1$, tandis que (10) garde sa validité même si

(*) Citation de M. L. SAUvaGE: Théorie générale des systèmes d'éruations différentielles linéaires et homogènes, p. 176; Paris, 1895. 
nous posons $p=q=1$; nous aurons dans ce cas cette formule particulière

$$
\gamma_{1,1}=\frac{1}{2}(\log 2)^{2}
$$

Pour déduire à l'aide de (a) des expressions analogues pour les séries $c_{p, q}$ et $\delta_{p, q}$ il faut considérer séparément le dernier terme des deux sommes figurant an second membre de cette formule; avec cette précaution le même procédé donnera ces deux formules analogues.

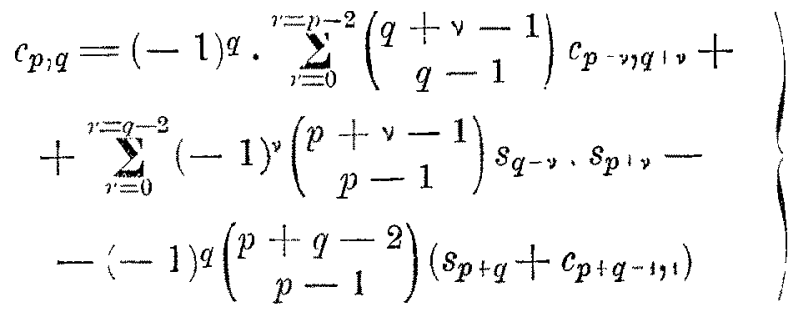

$$
\begin{aligned}
& \delta_{p, q}=(-1)^{q} \cdot \sum_{\nu=0}^{\gamma=p-2}\left(\begin{array}{c}
q+v-1 \\
q-1
\end{array}\right) d_{p-\nu, q+\nu}+ \\
& +\sum_{v=0}^{r=q-2}(-1)^{\nu}\left(\begin{array}{c}
p+y-1 \\
p-1
\end{array}\right) s_{q-\psi} \cdot \sigma_{p+\nu}+ \\
& +(-1)^{q}\left(\begin{array}{c}
p+q-2 \\
p-1
\end{array}\right)\left(\gamma_{p+q-1,1}-\sigma_{p+q}\right) ;
\end{aligned}
$$

dans le cas particulier $q=1$ il faut dans ces formules supprimer au second membre les sommes qui deviendront illusoires. Dans (12) il faut toujours admettre $p>1$, tandis que (13) donnera pour $p=1$ ce cas particulier

$$
\gamma_{q, 1}-(-1)^{q} s_{1, q}=\sigma_{q+1}-\sum_{\nu=0}^{r=q-2}(-1)^{\nu} s_{q-\nu} \cdot \sigma_{\nu+1} .
$$

III. Formules RÉcursives pour Les séries $s_{n}$ et $\sigma_{n}$.

Les formules plus générales que nous venons de développer nous fournissent un simple moyen pour déduire un nombre de formules récursives pour les séries numériques $s_{n}$ et $\sigma_{n}$, formule qui nous conduiront aux formules bien connues pour $s_{2 n}$ et $\sigma_{2 n}$.

Mettons en premier lieu dans (12) $q=1$, nous aurons généralement, 
pour $p>2$, cette formule élégante

$$
c_{p, 1}=s_{p+1}-\sum_{2}^{p-1} c_{p-p+1, p}
$$

et particulièrement pour $p=2$

$$
c_{2,1}=s_{3} \text {. }
$$

Écrivons maintenant dans la somme figurant au second membre de (14) les termes dans l'ordre invers puis ajoutons à (14) la formule ainsi obtenue, nous aurons, en vertu de (7), cette formule récursive

$$
\sum_{2}^{p-2} s_{\vartheta} s_{p-9+1}=p, s_{p+1}-2 c_{p, 1}
$$

posons ensuite dans (12) $p=2, q=2 n-2$, nous trouvons pour $n>1$

$$
(2 n-2)\left(c_{2 n-1}, 1+s_{2 n}\right)=\sum_{0}^{2 n-4}(-1)^{\nu}(\nu+1) s_{v+2} . s_{2 n-\nu-2},
$$

d'où, en écrivant dans l'ordre inverse les termes de la somme figurant au second membre, cette formule nouvelle

$$
2\left(c_{2 n-1,1}+s_{2 n}\right)=\sum_{1}^{n-1} s_{2 \nu} \cdot s_{2 n-2 \nu}-\sum_{1}^{n-2} s_{3 y+1} \cdot s_{2 n-2 \nu-1} .
$$

Cela posé, mettons dans (15) $p=2 n-1$, nous aurons immédiatement, en vertu de $\left(15^{\text {bis }}\right)$, ces deux formules

$$
\begin{aligned}
& \sum_{1}^{n-1} s_{2 y} \cdot s_{2 n-2 \nu}=\frac{2 n+1}{2} \cdot s_{n} \\
& \sum_{1}^{n-2} s_{2 y+1} \cdot s_{2 n-2 \nu-1}=\frac{2 n-3}{2} \cdot s_{2 n}-2 c_{2 n-1,1},
\end{aligned}
$$

dont la première est bien connue; on la dèmontre ordinairement en s'appuyant sur des propriétés fondamentales des fonctions trigonométriques.

Prenons maintenant pour point de départ la formule (11), et posons-y $q=1$, nous aurons

$$
d_{p, 1}=s_{p} \sigma_{1}-\sum_{0}^{p-1} \delta_{p-v, v+1}
$$

ce qui donnera, en vertu de (9) et en appliquant (8) pour $s_{p} \sigma_{1}$, cette autre formule récursive

$$
\sum_{1}^{p} \sigma_{y} \sigma_{p-y+1}=p s_{p+1}-2 \sigma_{p+1}+2 \gamma_{1, p}
$$


196 Niels Nielsen: Recherches sur le carré de la dérivée logarithmique

mettons ensuite dans (10) $p=1, q=2 n \cdot-1$, nous aurons de mème

$$
\sum_{\frac{1}{v}}^{2 n-2}(-1)^{r} \sigma_{r+1} \cdot \sigma_{2 n-r-1}=2 \gamma_{1,2 n-1},
$$

d'où, en vertu de (17) pour $p=2 n-1$,

$$
\begin{aligned}
& \sum_{1}^{n-1} \sigma_{2 n} \cdot \sigma_{2 n-2 r}=\frac{2 n-1}{2} \cdot s_{i n}-\sigma_{2 n} \\
& \sum_{0}^{n-1} \sigma_{2 r+1} \cdot \sigma_{2 n-2 r-1}=\frac{2 n-1}{2} \cdot s_{2 n}-\sigma_{2 n}+2 \gamma_{1,2 n-1},
\end{aligned}
$$

formules dont la première est bien connues comme une conséquence immédiate des propriétés fondamentales des fonctions trigonométriques.

Pour obtenir une troisième catégorie des formules récursives posons dans (10) et dans (13) $q=1$, nous aurons respectivement

$$
\begin{aligned}
& 2 \gamma_{p, 1}=\sigma_{p} \sigma_{1}-\sum_{0}^{p-2} \gamma_{p-r, r+1} \\
& \delta_{p, 1}+\gamma_{p, 1}=\sigma_{p+1}-\sum_{0}^{p-2} d_{p-r, r+1} ;
\end{aligned}
$$

soustrayons maintenant ces deux formules, puis appliquons (8) et transformons à l'aide de (9) le produit $\sigma_{p} \sigma_{1}$, nous aurons

$$
\gamma_{p},+\delta_{1, p}=\sum_{u}^{p-2} s_{p-r} \cdot \sigma_{r+1}+s_{p+1}-p \sigma_{p+1} .
$$

La formule $\left(13^{\text {bis }}\right)$ donnera de même, pour $q=2 n-1$, cette expression analogue

$$
\gamma_{2 n-1,1}+\delta_{1,2 n-1}=\sum_{0}^{2 n-3}(-1)^{r} \tau_{r+1} . s_{3 n \cdot r-1}+\sigma_{2 n},
$$

d'où, en vertu de (19) ces deux nouvelles formules réeursives

$$
\begin{aligned}
& \sum_{1}^{n-1} \sigma_{2 r} . s_{2 n-2 r}=n \sigma_{2 n}-\frac{1}{2} s_{2 n} \\
& \sum_{0}^{n-2} \sigma_{2 r+1} \cdot s_{2 n-2 n-1}=(n-1) \sigma_{2 n}-\frac{1}{2} s_{2 n}+\gamma_{2 n-3,1}+\delta_{2,2 n-1} .
\end{aligned}
$$

Considérons encore cette autre série numérique

$$
t_{n}=\frac{1}{2^{n}} \cdot s_{n}\left(\frac{1}{2}\right)=\frac{1}{1^{n}}+\frac{1}{3^{n}}+\frac{1}{5^{n}}+\frac{1}{7^{n}}+\cdots, \quad n>1,
$$


ou, ce qui vaut autant,

$$
t_{n}=\frac{1}{2}\left(s_{n}+\sigma_{n}\right)
$$

nous aurons de (16) et (20) ou de (18) et (20) respectivement ces deux autres formules récursives

$$
\begin{aligned}
& \sum_{1}^{n-1} t_{2 n} . s_{2 n-2 r}=n t_{2 n} \\
& \sum_{1}^{n-1} t_{2 r} \cdot \sigma_{2 n-2 r}=(n-1) t_{2 n},
\end{aligned}
$$

de façon qu'une addition de ces deux formules donnera cette autre

$$
\sum_{1}^{n-1} t_{2 r} \cdot t_{2 n-2 r}=\frac{2 n-1}{2} \cdot t_{2 n}
$$

IV. Applications auX fonctions $\left(s_{1}(x)\right)^{2}, s_{1}(x) \sigma_{1}(x)$ et $\left(\sigma_{1}(x)\right)^{2}$.

Les formules purement numériques que nous venons d'établir s'appliqueront immédiatement à l'évaluation de six produits formés de deux facteurs pris parmi ces quatre fonctions $s_{1}(x), s_{1}(1-x), \sigma_{1}(x)$ et $\sigma_{1}(1-x)$. Considérons d'abord le carré de $s_{!}(x)$, nous aurons, en vertu de (1 $\left.{ }^{\text {bis }}\right)$, et en appliquant la règle de Cauchy

$$
\left(s_{1}(1-x)\right)^{2}=\sum_{n=1}^{n=\infty}\left(s_{2} s_{n-2}+s_{3} s_{n-3}+\cdots+s_{n-2} s_{2}\right) x^{n-2}
$$

d'où, en vertu de $(15)$ et de $\left(14^{\text {bis }}\right)$,

$$
\left(s_{1}(1-x)\right)^{2}=s_{2}(1-x)-s_{2}-2 \cdot \sum_{n=2}^{n=\infty} c_{n, 1} x^{n-1} \text {. }
$$

Pour transformer ensuite la série infinie figurant au second membre de cette formule introduisons an lieu des $c_{n, 1}$ les séries infinies correspondantes, nous trouvons pour le coefficient du terme

$$
\frac{1}{1}+\frac{1}{2}+\frac{1}{3}+\cdots+\frac{1}{r-1}
$$

Annali di Malematica. Serie III. tomo IX. 
198 Niels Nielsen: Recherches sur le carré de la dérivée logarithmique

cette expression

$$
\frac{x}{r^{2}}+\frac{x^{2}}{r^{3}}+\frac{x^{3}}{r^{4}}+\cdots=\frac{x}{r(r-x)}=\frac{1}{r-x}-\frac{1}{r},
$$

et nous aurons finalement, après avoir posé $1-x$ au lieu de $x$, cette formule

$$
\left(s_{1}(x)\right)^{2}=s_{2}(x)-s_{2}+2 \xi(x),
$$

où nous aurons posé pour abréger

$$
\xi(x)=\sum_{n=1}^{n=\infty}\left(\frac{1}{x+n}-\frac{1}{n+1}\right)\left(\frac{1}{1}+\frac{1}{2}+\cdots+\frac{1}{n}\right) .
$$

La formule (23) n'est démontrée pour le moment que si $|x|<1$, il est vrai; or, les deux membres de cette formule représentent des fonctions holomorphes dans toute l'étendue du plan des $x$, à l'exception des points isolés $0,-1,-2,-3, \ldots ;$ c'est-à-dire que notre formule susdite est applicable pour une valeur quelconque de $x$.

Cherchons maintenant directement, d'après la règle de $\mathrm{C}_{A \mathrm{uchy}}$ le carré du second membre de (1), nous aurons, en vertu de (23), pour la fonction

$$
\rho(x)=\sum_{n=1}^{n=\infty}\left(\frac{1}{x+n}-\frac{1}{n+1}\right)\left(\frac{1}{x}+\frac{1}{x+1}+\cdots+\frac{1}{u+n-1}\right)
$$

cette autre expression remarquable

$$
\rho(x)=\sum_{n=0}^{n=\infty} \frac{1}{(x+n)(n+1)}-s_{2} .
$$

Prenons encore une fois comme point de départ la formule $\left(1^{\text {bis }}\right)$, nous aurons de la même manière, en appliquant $\left(15^{\text {bis }}\right)$, cette autre formule

$$
s_{1}(1+x) s_{1}(1-x)=-2 \cdot \sum_{n=2}^{n-\infty}\left(c_{2 n-1,1}+s_{2 n}\right) x^{2 n-2},
$$

de sorte que l'identité

$$
s_{1}(1+x)=s_{1}(x)-\frac{1}{x}
$$

donnera immédiatement

$$
s_{1}(x) s_{1}(1-x)=-2 \cdot \sum_{2}^{\infty} c_{2 n-1,1} x^{2 n-2}-\sum_{2}^{\infty}(-1)^{n} s_{n} x^{n \cdots 2}+2 s_{2},
$$

d'où, après une légère transformation, cette autre formule remarquable

$$
s_{1}(x) s_{1}(1-x)=2 s_{3}-\xi(x)-\xi(1-x)
$$


Pour trouver les formules correspondantes qui contiennent la fonction $\sigma_{1}(x)$ prenons pour point de départ la formule ( $4^{\text {bis }}$, nous trouvons immédiate. ment, en vertu de (17),

$$
\left(\sigma_{1}(1-x)\right)^{2}=\sum_{n=2}^{n=\infty}\left((n-1) s_{n}+2 \gamma_{1, n-1}-2 \sigma_{n}\right) x^{n-2},
$$

d'où, en posant $1-x$ au lieu de $x$, cette autre formule générale

$$
\left(z_{1}(x)\right)^{2}=s_{2}(x)-2 \eta(x),
$$

où nous arons posé pour abréger

$$
n(x)=\sum_{n=1}^{n=\infty} \frac{(-1)^{n-1}}{n}\left(\frac{1}{x}+\frac{1}{x+1}+\cdots+\begin{array}{c}
1 \\
x+n-1
\end{array}\right) .
$$

Appliquons maintenant la formule $\left(17^{\mathrm{bis}}\right)$, nous aurons de même

$$
\sigma_{1}(1+x) \sigma_{1}(1-x)=2: \sum_{1}^{\infty} y_{1}, n-1 \cdot x^{2 n-2},
$$

de sorte que l'identité

$$
\sigma_{1}(1+x)=\frac{1}{x} \cdot \sigma_{1}(x)
$$

donnera, après une légère transformation

$$
\sigma_{1}(x) \sigma_{1}(1-x)=n(x)+n(1-x) \text {. }
$$

Enfin, pour évaluer le produit $s_{1}(x) \sigma_{1}(x)$, appliquons ces deux identités

$$
\begin{gathered}
2 s_{1}(x)+2 \sigma_{1}=s_{1}\left(\frac{x}{2}\right)+s_{1}\left(\frac{x+1}{2}\right) \\
2 \sigma_{1}(x)=s_{1}\left(\frac{x}{2}\right)-s_{1}\left(\frac{x+1}{2}\right),
\end{gathered}
$$

nous aurons immédiatement, en vertu de (23),

$$
s_{1}(x) \sigma_{1}(x)=\sigma_{2}(x)-\sigma_{1} \cdot \sigma_{1}(x)-\frac{1}{2} \xi\left(\frac{x}{2}\right)+\frac{1}{2} \xi\left(\frac{x+1}{2}\right) .
$$

De la même manière nous aurons, à l'aide de (13 $\left.{ }^{\text {bis }}\right)$, cette dernière formule de ce genre

$$
s_{1}(x) \sigma_{1}(1-x)=\sigma_{1} \cdot \sigma_{1}(x)-\xi_{1}(1-x)+\xi_{2}(x),
$$

où nous avons posé pour abréger

$$
\left.\begin{array}{l}
\xi_{1}(x)=\sum_{n=1}^{n=\infty} \frac{(-1)^{n-1}}{n+x}\left(\frac{1}{1}+\frac{1}{2}+\frac{1}{3}+\cdots+\frac{1}{n}\right) \\
\varkappa_{2}(x)=\sum_{n=1}^{n=\infty} \frac{(-1)^{n-1}}{n+x}\left(\frac{1}{1}-\frac{1}{2}+\frac{1}{3}-\cdots+\frac{(-1)^{n-1}}{n}\right)
\end{array}\right\}
$$


200 Niels Nielsen: Recherches sur le carré de la dërivée logarithmique

Certainement, les six produits que nous venons d'évaluer nous fournissent un simple moyen pour généraliser beaucoup les formules numériques données dans la section III. Différentions par exemple $(n-2)$ fois par rapport à $x$ la formule (23), nous aurons

$$
\begin{gathered}
\sum_{1}^{n-1} s_{r}(x) s_{n-r}(x)=(n-1) s_{n}(x)- \\
-2 \cdot \sum_{r=1}^{r=1} \frac{1}{(x+r)^{n-1}}\left(\frac{1}{1}+\frac{1}{2}+\frac{1}{3}+\cdots+\frac{1}{r}\right),
\end{gathered}
$$

formule qui, pour $x=1$, donnera immédiatement la relation (15).

De la même manière nous obtenons de (26) et de (27) ces deux autres formules

$$
\begin{gathered}
\sum_{1}^{n} \sigma_{r}(x) \sigma_{n-r}(x)=(n-1) s_{n}(x)-\frac{(-1)^{n} \cdot 2}{(n-2) !} \cdot D_{x}^{n-2} \eta(x) \\
\sum_{1}^{n-1}(-1)^{r-1} \sigma_{n-r}(x) \sigma_{r}(1-x)=\frac{(-1)^{n}}{(n-2) !}\left(D_{x}^{n-2} \eta(x)+D_{x}^{n-2} \eta(1-x)\right)
\end{gathered}
$$

posons dans ces formules $x=\frac{1}{2}$ et $2 n$ au lieu de $n$, nous aurons pour cette série numérique

$$
\tau_{n}=\frac{1}{2^{n}} \cdot \sigma_{n}\left(\frac{1}{2}\right)=\frac{1}{1^{n}}-\frac{1}{3^{n}}+\frac{1}{5^{n}}-\frac{1}{7^{n}}+\cdots, \quad n \geqq 1
$$

cette formule récursive

$$
\sum_{0}^{n-1} \tau_{2 n+1} \cdot \tau_{2 n-2,-1}=\left(n-\frac{1}{2}\right) t_{2 n} .
$$

Remarquons encore que (28) et (29) donnent respectivement ces deux autres formules

$$
\begin{aligned}
\sum_{1}^{n-1} s_{r}(x) \sigma_{n-r}(x)=(n-1) \sigma_{n}(x)-\sigma_{1}, \sigma_{n-1}(x)- & \\
& -\frac{(-1)^{n}}{2(n-2) !}\left(D_{x}^{n-2} \xi\left(\frac{x}{2}\right)-D_{s}^{n-2} \xi\left(\frac{x+1}{2}\right)\right) \\
\sum_{1}^{n-1}(-1)^{r-1} s_{n-r}(x) \sigma_{r}(1-x) & =\sigma_{1} \cdot \sigma_{n-1}(x)- \\
& -\frac{(-1)^{n}}{(n-2) !}\left(D_{x}^{n-2} \xi_{1}(1-x)-D_{x}^{n-2} \xi_{2}(x)\right)
\end{aligned}
$$


nous aurons cette autre relation numérique

$$
\sum_{0}^{n-1} \tau_{2 r+1}, t_{2 n-2 r}=n, \tau_{2 n+1} .
$$

L'analogie entre les formules que nous venons d'indiquer et celles de la section III est évidente; cependant, l'essay de trouver pour $s_{n}(x)$ ou $o_{n}(x)$ des formules récursives analogues à $(16),(18)$ et (20) doit être désigné à l'avance comme infructueux. En effet, M. HöLder $\left(^{*}\right)$ a démontré que la fonction de Gauss $\Psi(x)$, ou ce qui revient au même, la fonction $s_{1}(x)$ ne peut satisfaire à aucune équation différentielle algébrique, et M. STadian (**) a démontré récemment, par une méthode analogue, que la fonction

$$
s_{1}\left(\begin{array}{c}
x \\
2
\end{array}\right)+s_{1}\left(\frac{1-x}{2}\right)
$$

possède la même proprièté; de plus, il et évident que la même démonstration peut être appliquèe à la fonction

aussi.

$$
2 \sigma_{1}(x)=s_{1}\left(\frac{x}{2}\right)-s_{1}\left(\frac{1+\infty}{2}\right)
$$

V. Expressions intégrates des six produits de deUX FONCTIONS

$$
s_{1}(x) \text { oU } \sigma_{1}(x) \text {. }
$$

Pour établir, à l'aide des formules de la section IV, des expressions intégrales pour les six produits de deux fonctions $s_{1}(x)$ ou $\sigma_{1}(x)$ appliquons cette méthode générale: Supposons égal à un au moins le rayon de convergence de cette série de puissances

$$
f(t)=a_{3}+a_{1} t+a_{2} t^{2}+a_{3} t^{3}+\cdots,
$$

et supposons de plus que cette série

$$
\frac{a_{0}}{1}+\frac{a_{1}}{2}+\frac{a_{2}}{3}+\frac{a_{3}}{4}+\cdots
$$

(*) Mathematische Amalen, t. 28, p. 7 .

(**) Ein Satz über Funktionen, die algebraisehe Differentialgleichungen befriedigen, p. 32, (Thèse de doctorat, Helsingfors, 1902). 
202 Niels Nielsen: Recherches sur le carré de la dérivée logarithmique

nous aurons, en appliquant un théorème bien connu (*), cette formule fondamentale dans les recherches qui nous occupent ici

$$
\int_{0}^{1} f(t) t^{x-1} d t=\frac{a_{0}}{x}+\frac{a_{1}}{x+1}+\frac{a_{2}}{x+2}+\cdots, \quad \Re(x)>0 .
$$

De plus, il est clair que la série infinie figurant au second membre de $\left(31^{\text {bis }}\right)$ est convergente dans toute l'étendue du plan des $x$, à l'exception des points isolés $0,-1,-2,-3, \ldots$, où un des termes de la série susdite deviendra infini.

Pour appliquer maintenant à la fonction $\xi(x)$, définie par $\left(23^{\text {bis }}\right)$, notre méthode générale, il s'agit d'étudier cette série de puissances

$$
f(t)=\frac{1}{1} t+\left(\frac{1}{1}+\frac{1}{2}\right) t^{2}+\left(\frac{1}{1}+\frac{1}{2}+\frac{1}{3}\right) t^{3}+\cdots, \quad|t|<1
$$

ce qui donnera immédiatement

$$
f(t)=\frac{1}{1} \cdot \frac{t}{1-t}+\frac{1}{2} \cdot \frac{t^{2}}{1-t}+\frac{1}{3} \cdot \frac{t^{3}}{1-t}+\cdots=-\frac{1}{1-t} \cdot \log (1-t),
$$

de sorte que nous obtenons cette expressions intégrale

$$
\xi(x)=-\int_{0}^{1} \frac{\left(t^{x-1}-1\right) \log (1-t)}{1-t} d t, \quad \Re(x)>0
$$

d'où, en vertu de $\left(3^{\text {bis }}\right)$ et $(23)$,

$$
\left(s_{1}(x)\right)^{2}=\int_{0}^{1} \frac{\left(t^{x-1}-1\right)(2 \log (1-t)-\log t)}{1-t} d t, \quad \Re(x)>0
$$

tandis que (25) donnera de même cette expression correspondante

$s_{1}(x) s_{1}(1-x)=\int_{0}^{1} \frac{\left(t^{x-1}+t-x-2\right) \log (1-t)-\log t}{1-t} d t, \quad 2>\Re(x)>0$

$\left(^{*}\right)$ Voir M. DrNx: Grundlagen für eine Theorie der Functionen einer veränderlichen reellen Grösse, p. 525; Leipsic, 1892. 
enfin les formules $\left(6^{\text {bis }}\right)$ et $(22)$ donneront cette troisième formule analogue

$$
\begin{gathered}
s_{1}(x) \sigma_{1}(x)=\frac{1}{2} \cdot \int_{0}^{1 t^{\frac{x}{2}-1}-\frac{\left.t^{\frac{x-1}{2}}\right)}{1-t} \log (1-t)} d t- \\
-\int_{0}^{1} \frac{t^{x-1} \log (2 t)}{1+t} d t, \quad \because(x)>0 .
\end{gathered}
$$

Considerons ensuite la fonction $n(x)$, nous trouverons cette série de puissances

on bien

$$
f(t)=\log 2+\left(\log 2-\frac{1}{1}\right) t+\left(\log 2-\frac{1}{1}+\frac{1}{2}\right) t^{2}+\cdots
$$

$f(t)==\left(1+t+t^{2}+\cdots\right) \log 2-\left(t+t^{3}+\cdots\right)_{1}^{1}+\left(t^{2}+t^{3}+\cdots\right) \frac{1}{2}-\cdots$, ce qui donnera

$$
f(t)=\frac{\log 2-\log (1+t)}{1-t}
$$

d'où cette expression intégrale

$$
n(x)=\int_{0}^{1} \frac{\log 2-\log (1+t)}{1-t} \cdot t^{x-1} d t, \quad \Re(x)>0
$$

et nous obtenons par suite, en vertu de $\left(3^{\text {bis }}\right)$ et de $(26)$,

$$
\left(\sigma_{1}(x)\right)^{2}=\int_{0}^{1} \frac{2 \log (1+t)-2 \log 2-\log t}{1-t} \cdot t^{x+1} d t, \quad \Re(x)>0,
$$

tandis que (27) donnera cette formule analogue

$$
\sigma_{1}(x) \sigma_{1}(1-x)=\int_{0}^{1} \frac{(\log 2-\log (1+t))\left(t^{x-1}+t^{-x}\right)}{1-t} d t, \quad 1>\Re(x)=0 .
$$

Nous avons encore à étudier les deux fonctions $\xi_{1}(x)$ et $\xi_{2}(x)$; considérons d'abord $\xi_{1}(x)$, la fonction $f(t)$ correspondante se détermine à l'aide de cette série

$$
f(t)=\frac{1}{1} \cdot t-\left(\frac{1}{1}+\frac{1}{2}\right) \cdot t^{2}+\left(\frac{1}{1}+\frac{1}{2}+\frac{1}{3}\right) t^{3}-\cdots ;
$$


c'est-à-dire que nous aurons

$$
f(t)=\frac{1}{1} \cdot \frac{t}{1+t}-\frac{1}{2} \cdot \frac{t^{2}}{1+t}+\frac{1}{3} \cdot \frac{t^{3}}{1+t}-\cdots=\frac{\log (1+t)}{1+t},
$$

de sorte que nous obtenons

$$
\xi_{1}(x)=\int_{0}^{1} \frac{\log (1+t)}{1+t} \cdot t^{x-1} d t, \quad \Re(x)>-1 .
$$

Quant à la fonction $\xi_{2}(x)$, nous aurons

ou bien

$$
f(t)=\frac{1}{1} t-\left(\frac{1}{1}-\frac{1}{2}\right) t^{2}+\left(\frac{1}{1}--\frac{1}{2}+\frac{1}{3}\right) t^{3}-\cdots
$$

$$
f(t)=\frac{t}{1} \cdot \frac{1}{1+t}+\frac{t^{2}}{2} \cdot \frac{1}{1+t}+\cdots=-\frac{\log (1+t)}{1+t}
$$

ce qui donnera

$$
\xi_{2}(x)=-\int_{0}^{1} \frac{\log (1-t)}{1+1} \cdot t^{x-1} d t, \quad \Re(x)>-1 .
$$

Cela posé, la formule (29) donnera, en vertu de $\left(6^{\text {bis }}\right)$,

$$
\begin{gathered}
s_{1}(x) \sigma_{1}(1-x)= \\
=\int_{0}^{1} \frac{(\log 2+\log (1-t)) t^{x-1}-\log (1+t) \cdot t^{-x}}{1+t} d t, \quad 2>\Re(x)>0 .
\end{gathered}
$$

Enfin, nous avons à étudier la fonetion $\rho(x)$ définie par $\left(24^{\text {bis }}\right)$, ce qui nous conduira à cette fonction auxiliaire

$$
f(t)=\frac{1}{1}+\frac{1}{2} t+\frac{1}{3} t^{2}+\frac{1}{4} t^{3}+\cdots=-\frac{\log (1-t)}{t},
$$

d'où, nous obtiendrons, en vertu de $\left(3^{\text {bis }}\right)$,

$$
\rho(x)=\int_{0}^{1}\left(\frac{\log t}{1-t}-\log (1-t) \cdot t^{x-2}\right) d t, \quad \Re(x)>0 .
$$


VI. Développements en sḱries dF Factorieties.

Les expressions intégrales que nous venons de dérelopper pour nos functions nous foumissent un moyen infaillible pour décider si une tello fonction est développable ou non en série de factorielles de cette forme

$$
\Omega(x)=\frac{b_{0}}{x}+\frac{1 ! b_{1}}{x(x+1)}+\frac{2 ! b_{2}}{x(x+1)(x+2)}+\cdots,
$$

où les coefficients $b$ sont indépendants de $x$, et de plus les mêmes intégrales nous permettent de déduire ce développement, s'il est possible.

En effet, dans un mémoire récent $\left({ }^{*}\right)$ j'ai démontré que la condition suffisante et nócessaire qui doit être remplie par une fonction développable en série de la forme (a) est que la fonction en question se présente sous cette forme

$$
\Omega(x)=\int_{0}^{1} f(t) \cdot t^{x-1} d t
$$

où la fonction $f(t)$ doit être holomorphe aux environs du point $t=1$ et cela de sorte que $l_{i} ;$ série de puissances

$f(1-t)=b_{0}+b_{1} t+b_{2} t^{2}+b_{3} t^{3}+\cdots, \quad f^{(n)}(1)=(-1)^{n} n ! b_{n}$,

a son rayon de convergencé égal à un au moins, et, de plus, cette fonction $f(t)$ doit satisfaire à quelques autres conditions faciles à indiquer mais sans influence sur la question qui nous occupe ici.

Cela posé, on verra sur-le-champ que la condition (y) est en défaut pour $\xi(x), \xi_{2}(x)$ et $\rho(x)$ et pour toutes leurs dérivées, parce que les fonctions correspondantes $f(t)$ possèdent en $t=1$ un point critique.

Pour développer en série de factorielles la fonction $\eta(x)$, écrivons sous cette forme l'expression intégrale (36)

$$
n(x)=-\int_{0}^{1} \frac{\log \left(1-\frac{t}{2}\right)}{t}(1-t)^{x-1} d t=\sum_{s=1}^{s=\infty} \frac{1}{s \cdot 2^{s}} \cdot \int_{0}^{1} t^{s-1}(1-t)^{x-1} d t
$$

(*) Annales de d'Ecole Normale. 3. ${ }^{\mathrm{e}}$ série, t. 19, p. 419. Paris, 100\%. 
ce qui donnera immédiatement cette série de factorielles

$$
n(x)=\sum_{s=1}^{s=\infty} \frac{(s-1) !}{s \cdot 2^{s}} \cdot \frac{1}{x(x+1) \ldots(x+s-1)}
$$

qui est valable dans toute l'étendue du plan des $x$.

Nous aurors de la même manière, en vertu de $\left(3^{\text {bis }}\right)$, cet autre développement

$$
s_{2}(x)=\sum_{s=1}^{s=\infty} \frac{(s-1) !}{s} \cdot \frac{1}{x(x+1) \ldots(x+s-1)}, \quad \Re(x)>0,
$$

d'où, en vertu de (26),

$$
\left(\sigma_{1}(x)\right)^{2}=\sum_{s=2}^{s=\infty} \frac{2^{s-1}-1}{s \cdot 2^{s-1}} \cdot \frac{1}{x(x+1) \ldots(x+s-1)}, \quad \Re(x)>0 .
$$

Quant à la fonction $\xi_{1}(x)$, il s'agit de déterminer ce coefficient

$$
f^{(x)}(2), \quad \text { où } \quad f(t)=\frac{\log t}{t}
$$

or, un calcul direct donnera, à l'aide de la formule de Leibnitz,

$$
f^{(n)}(t)=\frac{(-1)^{n} n !}{t^{n+1}}(\log t-\lambda(n)),
$$

où nous avons posé pour abréger $\lambda(0)=0$ et généralement pour $n \geq 1$

$$
\lambda(n)=\frac{1}{1}+\frac{1}{2}+\cdots+\frac{1}{n}
$$

cela posé, les formules générales $(\alpha)$ et $(\gamma)$ donnent jci

$$
\xi_{1}(x)=\sum_{s=0}^{\infty} \frac{s !}{2^{s+1}} \cdot \frac{\log 2-\lambda(s)}{x(x+1) \ldots(x+s)},
$$

développement qui est valable dans toute l'étendue du plan des $x$. Remarquons que nous aurons

$$
\sigma_{1}(x)=\sum_{s=0}^{s=\infty} \frac{s !}{2^{s+1}} \cdot \frac{1}{x(x+1) \ldots(x+s)},
$$

la formule (46) s'écrira sous cette forme aussi

$$
\sigma_{1}(x) \log 2-\xi_{1}(x)=\sum_{s=1}^{s=\infty} \frac{s !}{2^{s+1}} \cdot \frac{\lambda(s)}{x(x+1) \ldots(x+s)} \cdot
$$

Considérons maintenant les séries de factorielles d'une forme entièrement 
différente de $(\alpha)$ que M. Pincherle $\left(^{*}\right)$ a étudiées récemment. Pour l'intégrale ( $\beta$ ) on obtiendra de tels développements en posant dans cette intégrale $1-t$ au lieu de $t$, ce qui donnera

$$
\Omega(1-x)=\int_{0}^{1} f(1-t)(1-t)^{-\infty} d t^{\varepsilon}
$$

cela posé, supposons intégrable de $t=0$ à $t=1$ la fonction $f(1-t)$, une application de la formule du binome donnera immédiatement ce developpement

$$
\Omega(1-x)=\sum_{s=0}^{s=\infty} a_{s}\left(\begin{array}{l}
x \\
s
\end{array}\right),
$$

où nous avons posé pour abréger

$$
a_{s}=\int_{0}^{1} f(1-t) t^{s} d t
$$

L'application de ces formules générales aux fonctions particulières que nous venons d'introduire est évidente; considérons d'abord $\xi(1-x)$, nous aurons immédiatement

ce qui donnera

$$
a_{0}=0 \quad a_{s}=-\int_{0}^{1} t^{s-1} \log t d t=\frac{1}{s^{2}},
$$

$$
\xi(1-x)=\sum_{s=1}^{s=\infty} \frac{1}{s^{2}} \cdot\left(\begin{array}{l}
x \\
s
\end{array}\right), \quad \Re(x)<1,
$$

tandis que nous aurons pour $s_{2}(1-x)$ ces coefficients

$$
a_{s}=-\int_{0}^{1} t^{s-1} \log (1-t) d t=\sum_{n=1}^{n=\infty} \frac{1}{n(n+s)},
$$

ou bien

$$
a_{0}=\frac{\pi^{2}}{6}, \quad a_{s}=\frac{1}{s} \cdot \sum_{n=1}^{n=\infty}\left(\frac{1}{n}-\frac{1}{n+s}\right)=\frac{1}{s} \cdot \lambda(s),
$$

de facon que nous obtiendrons

$$
s_{2}(1-x)=\frac{\pi^{2}}{6}+\sum_{s=1}^{s=\infty} \frac{\lambda(s)}{s} \cdot\left(\begin{array}{c}
x \\
s
\end{array}\right), \quad \Re(x)<1 .
$$

(*) Rendiconti della reale Accademia dei Lincei, serie 5. ${ }^{2}, \mathrm{t}, 11$, p. 139, p. 417; 1902. 
208 Niels Nielsen: Recherches sur le carré de la dérivée logarithmique

Ces deux développentents trouvés, nous aurons immédiatement, en vertu de (23), cet autre

$$
\left(s_{1}(1-x)\right)^{2}=\sum_{s=1}^{s=\infty} \frac{1}{s}\left(\lambda(s)-\frac{2}{s}\right) \cdot\left(\begin{array}{l}
x \\
s
\end{array}\right) .
$$

Quant à la fonction $n(1-x)$, nous aurons

$$
a_{s}=-\int_{0}^{1} \log \left(1-\frac{t}{2}\right) t^{s-1} d t=\sum_{n=1}^{n=\infty} \frac{1}{n(n+s)} \cdot \frac{1}{2^{n}},
$$

d'où, eu vertu d'une formule de LEGENDRE $\left({ }^{*}\right)$,

$$
a_{0}=\sum_{n=1}^{n=\infty}-\frac{1}{n^{2}} \cdot \frac{1}{2^{n}}=\frac{\pi^{2}}{12}-\frac{1}{2}(\log 2)^{2}
$$

et plus généralement pour $s \geq 1$

$$
a_{s}=\frac{1}{s} \cdot \sum_{n=1}^{n=\infty}\left(\frac{1}{n}-\frac{1}{n+s}\right) \cdot \frac{1}{2^{n}}=\frac{2^{s}}{s} u(s)-\frac{2^{s}-1}{s} \log 2,
$$

où nous avons posé pour abréger $\mu(1)=0$ et, pour $s \geq 1$,

$$
\mu(s)=\frac{1}{1} \cdot \frac{1}{2}+\frac{1}{2} \cdot \frac{1}{2^{2}}+\cdots+\frac{1}{s} \cdot \frac{1}{2^{s}} .
$$

Cela posé, nous aurons

$$
n(1-x)=\frac{\pi^{2}}{12}-\frac{1}{2}(\log 2)^{2}+\sum_{s=1}^{s=\infty} \frac{1}{s}\left(2^{s} \mu(s)-\left(2^{s}-1\right) \log 2 \cdot\left(\begin{array}{l}
x \\
s
\end{array}\right),\right.
$$

où, en vertu de (26) et (48), cet autre développement

$$
\begin{gathered}
\left(\sigma_{1}(1-x)\right)^{2}=(\log 2)^{2}+ \\
+\sum_{s=1}^{\sim=\infty} \frac{1}{s}\left(\lambda(s)-2^{s+1} \mu(s)+\left(2^{s+1}-2\right) \log 2\right) \cdot\left(\begin{array}{l}
x \\
s
\end{array}\right) .
\end{gathered}
$$

Le développement correspondant pour $\sigma_{1}(1-x)$ deviendra plus compliqué; pour la fonction $\xi_{2}(1-x)$ nous trouvons, au contraire, ce coefficient général

$$
a_{s}=-\int_{0}^{1} \frac{\log t}{2-t} \cdot t^{s} d t=\sum_{n=1}^{n=\infty} \frac{1}{2^{n}} \cdot \frac{1}{(n+s)^{2}},
$$

(*) Exercices de calcul différentiel et intégral, t. II, p. 244. 
d'où, en vertu de la formule de Legrndre,

$$
a_{s}=2 s\left(\frac{\pi^{2}}{12}-\frac{1}{2}(\log 2)^{2}-\mu_{1}(s)\right)
$$

oì nous avons posé pour abréger $\mu_{1}(0)=0$ et plus généralement, pour $s \geq 1$,

$$
\mu_{1}(s)=\frac{1}{1^{2}} \cdot \frac{1}{2}+\frac{1}{2^{2}} \cdot \frac{1}{2^{2}}+\frac{1}{3^{2}} \cdot \frac{1}{2^{3}}+\cdots+\frac{1}{s^{2}} \cdot \frac{1}{2^{s}},
$$

ce qui donnera

$$
\xi_{2}(1-x)=\sum_{s=0}^{s=\infty} 2^{s}\left(\frac{\pi^{2}}{12}-\frac{1}{2}(\log 2)^{2} \cdots-\mu_{1}(s)\right) \cdot\left(\begin{array}{l}
x \\
s
\end{array}\right)
$$

Considérons maintenant la dernière des fonctions particulières que nous avons introduites, savoir la fonction $\rho(1-x)$, nous aurons sans peine ce développement nouveau

$$
\rho(1-x)=\sum_{s=1}^{s=\infty}\left(\frac{\pi^{2}}{6}-\frac{1}{1^{2}}-\frac{1}{2^{2}} \cdots \cdots-\frac{1}{s^{2}}\right) \cdot\left(\begin{array}{c}
x \\
s
\end{array}\right) .
$$

En terminant ces recherches nous avons encore à donner quelques développements qui contiennent des séries de factorielles et du premier et du second genre. En premier lieu, la formule (27) donnera, en vertu de (43) et (50), ce développement

$$
\left.\begin{array}{c}
\sigma_{1}(x) \sigma_{1}(1-x)=\sum_{s=1}^{s=n} \frac{(s-1) !}{s \cdot 2^{s}} \cdot \frac{1}{x(x+1) \ldots(x+s-1)}+ \\
+\frac{\pi^{2}}{12}-\frac{1}{2}(\log 2)^{2}+\sum_{s=1}^{s=\infty} \frac{1}{s}\left(2 s \mu(s)-\left(2^{s}-1\right) \log 2\right) \cdot\left(\begin{array}{l}
x \\
s
\end{array}\right) .
\end{array}\right\}
$$

Écrivons encore sous cette forme la formule (29)

$$
s_{1}(1-x) \sigma_{1}(x)=\sigma_{1} \cdot \sigma_{1}(1-x)-\xi_{1}(x)+\xi_{2}(1-x) .
$$

il s'agit d'abord de développer la fonction $\sigma_{1}(1-x)$; or, nous troupons, en vertu de $(6)$,

$$
a_{s}=\int_{0}^{1} \frac{t^{s}}{2-t} d t=\sum_{n=1}^{n=\infty} \frac{1}{2^{n}} \cdot \frac{1}{s+n}=2^{s}(\log 2-\mu(s)),
$$

d'où

$$
\sigma_{1} \cdot \sigma_{1}(1-x)=\sum_{s=0}^{s} 2^{\infty}\left((\log 2)^{2}-\mu(s) \log 2\right) \cdot\left(\begin{array}{l}
x \\
s
\end{array}\right)
$$


210 Niels Nielsen: Recherches sur le carré de la dérivée logarithmique etc.

de sorte que nous obtiendrons finalement, en vertu de (46) et (52), ce développement

$$
\begin{aligned}
s_{1}(1-x) \sigma_{1}(x)= & \left.\sum_{s=0}^{s=\infty} 2^{s}\left(\frac{\pi^{2}}{12}+\frac{1}{2}(\log 2)^{2}-\mu(s) \log 2-\mu_{1}(s)\right) \cdot\left(\begin{array}{l}
x \\
s
\end{array}\right)-\right\} \\
& -\sum_{s=0}^{s=\infty} \frac{s !}{2^{s+1}} \cdot \frac{\log 2-\lambda(s)}{x(x+1) \ldots(x+s)} .
\end{aligned}
$$

Copenhague, le 19 avril 1903. 